\title{
Phonon emission in germanium and silicon by electrons and holes in applied electric field at low temperature
}

\author{
G. Wang ${ }^{\text {a) }}$ \\ Department of Physics, California Institute of Technology, Pasadena, California 91125, USA
}

(Received 16 September 2009; accepted 7 February 2010; published online 6 May 2010)

\begin{abstract}
The cryogenic dark matter search employs $\mathrm{Ge}$ and $\mathrm{Si}$ detectors to search for weakly interacting massive particle dark matter via its elastic-scattering interactions with nuclei while discriminating against interactions of background particles. These detectors distinguish nuclear recoils from electron recoils by simultaneously measuring phonon and ionization production in semiconducting substrates at sub-kelvin temperatures. They also reconstruct event position by quadrant-segmented measurement of the phonon signal. The ionization drift field does work on the electrons and holes. The charge carriers radiate this energy as acoustic phonons. At the typical applied field of $300 \mathrm{~V} / \mathrm{m}$ in $\mathrm{Ge}(400 \mathrm{~V} / \mathrm{m}$ in $\mathrm{Si})$, we self-consistently model the behavior of the electrons and holes using independent drifted Maxwellian distributions, each characterized by an average drift velocity and an effective temperature, and including acoustic phonon emission. We calculate the phonon power angular and frequency distributions. We find that the bias polarity affects these distributions and, therefore, the phonon collection efficiency in Ge. (C) 2010 American Institute of Physics. [doi:10.1063/1.3354095]
\end{abstract}

\section{INTRODUCTION}

Weakly interacting massive particles (WIMPs) are a well-motivated candidate for the dark matter in the universe [see, for e.g., (Refs. 1-5)]. The cryogenic dark matter search (CDMS) employs Ge and Si detectors to search for WIMPs via their elastic-scattering interactions with nuclei while discriminating against interactions of background particles. ${ }^{6-8}$ The CDMS detectors ${ }^{9,10}$ are disks of semiconducting Ge or $\mathrm{Si}, 7.6 \mathrm{~cm}$ in diameter and $1 \mathrm{~cm}$ thick, cooled to $\sim 40 \mathrm{mK}$. Ionizing radiation produces electrons and holes together with primary phonons. The charge carriers are drifted using an electric field, typically $300 \mathrm{~V} / \mathrm{m}$ in $\mathrm{Ge}(400 \mathrm{~V} / \mathrm{m}$ in $\mathrm{Si})$, and collected at two concentric electrodes on the flat face at which the bias potential is applied. Acoustic phonons, which are called Neganov-Luke phonons in this paper after the author names in Refs. 11 and 12, are produced during drift, and recombination phonons are emitted when the carriers reach the electrodes. Before these various types of phonons thermalize, they are absorbed in superconducting aluminum films on the detector surface and the resulting quasiparticles sensed by tungsten transition-edge sensors at the edges of these films. Each quadrant of the other (grounded) flat face is covered by such a thin-film circuit. The phonon signal, corrected for Neganov-Luke phonon emission, measures the recoil energy of each event. The relative delay of the phonon signal at the four phonon sensors and its partition among them provide a transverse position estimate. The ratio of ionization production to recoil energy (ionization yield) discriminates nuclear recoils from electron recoils with a rejection of better than $10^{4}$. Electron recoils within $\sim 10 \mu \mathrm{m}$ of the surface suffer suppressed ionization yield, sufficient to

\footnotetext{
${ }^{a)}$ Present address: Materials Science Division, Argonne National Laboratory, 9700 S Cass Ave., Lemont, IL 60439, USA. Electronic mail: gwang@anl.gov.
}

misclassify such events as nuclear recoils. Such events also have shorter phonon rise time and delay relative to the prompt ionization pulse, allowing us to reject more than $99 \%$ of such surface events.

Phonons produced in CDMS detectors fall into three categories: primary phonons, recombination phonons, and Neganov-Luke phonons. We consider each of these in turn, focusing on the effective size of the emission region and the emitted angular distribution.

Primary phonons are those created directly by the recoiling particle or by hot charge carriers as they thermalize. Primary phonons are very energetic and thus suffer substantial isotopic scattering that prevents them from propagating $\left(\right.$ rate $\left.\propto \nu^{4}\right){ }^{13,14}$ They must first downconvert via anharmonic decay $\left(\text { rate } \propto \nu^{5}\right)^{13}$ from the optical branch to the acoustic branch. Acoustic phonons have a mean free path larger than the detector thickness and are thus able to propagage quasiballistically. The timescale for the decay process to $1 \mathrm{THz}$ acoustic phonons is a few microseconds, during which time they diffuse over a distance of about $1 \mathrm{~mm} .{ }^{15}$ Because of Umklapp processes, ${ }^{16}$ no information about incident particle direction is preserved and thus the primary phonons may be treated as an isotropically emitting pointlike source.

Recombination phonons are generated when electrons and holes recombine in the electrodes, releasing the semiconductor gap energy. Because of the short carrier drift time across the detector $(\ll 1 \mu \mathrm{s})$, the carriers do not suffer substantial transverse diffusion and thus recombination phonons are emitted from approximately pointlike sources on each face. Additionally, the high impurity and defect densities at the surfaces randomize phonon emission direction.

As electrons and holes drift across the detector, they quickly accelerate to a constant velocity at which the work done on them by the externally applied electric field is balanced by emission of so-called Neganov-Luke phonons. In 
Sec. III, we adopt a drifted Maxwellian distribution for the carriers with an effective temperature and average drift velocity calculated self-consistently by requiring the emitted energy be equal to the work done by the electric field. In Sec. $\mathrm{IV}$, we then calculate the angular and frequency distributions of the emitted phonons at typical CDMS detector operating fields.

Because primary and recombination phonons are emitted isotropically and in a fashion that is independent of applied field magnitude or polarity (assuming the field is large enough to drift all carriers without trapping), this analysis focuses on the angular and frequency distributions of Neganov-Luke phonons and their impact on phonon collection efficiency in CDMS detectors as a function of field polarity.

\section{ELECTRON DEFORMATION POTENTIAL INTERACTION}

Near the band edges in semiconductors with parabolic band structures, the electron (hole) velocity $\vec{v}$ and wave vector $\vec{k}$ are related by

$$
m \vec{v}=\hbar \vec{k}
$$

and the electron (hole) energy $\epsilon$ and wave vector $\vec{k}$ are related by

$$
\epsilon=\frac{\hbar^{2} \vec{k}^{2}}{2 m}
$$

where $m$ is the electron (hole) effective mass. For $\mathrm{Si}$ and $\mathrm{Ge}$, these equations are reasonable approximations for electrons (holes) with energies near the bottom of the conduction band (top of the valence band). ${ }^{17}$

A carrier of wave vector $\vec{k}$ may suffer electron-phonon scattering via the deformation potential interaction, causing it to emit a phonon with wave vector $\vec{q}$. Momentum conservation yields

$$
\hbar \vec{k}=\hbar \vec{k}^{\prime}+\hbar \vec{q},
$$

where $\vec{k}^{\prime}$ is the electron wave vector after phonon emission. Energy conservation implies

$$
\frac{\hbar^{2} \vec{k}^{2}}{2 m}=\frac{\hbar^{2} \vec{k}^{2}}{2 m}+h \nu,
$$

where $\nu$ is the phonon frequency.

In calculating the angular distribution of emitted phonons, we work in a coordinate system in which the $z$-axis is the mean carrier drift direction (parallel or antiparallel to the applied field). We use a spherical coordinate system in which the polar and azimuthal angles of the carrier wave vector are $\alpha$ and $\beta$ and of the emitted phonon are $\chi$ and $\delta$. The angle between the carrier and phonon wave vectors is $\theta$. See Fig. 1. Because of the low carrier velocity (calculated below), Neganov-Luke phonons have frequencies less than 1 $\mathrm{THz}$ and thus may be assumed to be low on the acoustic branch and thus have a linear dispersion relation:

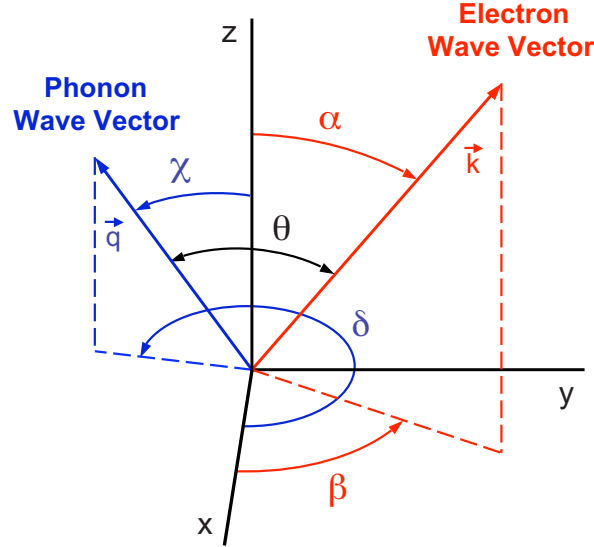

FIG. 1. (Color online) Diagram of electron and phonon wave vectors.

$$
\nu=\frac{s q}{2 \pi}
$$

where $s$ is the sound speed and $q$ is the magnitude of the phonon wave vector.

From energy conservation [Eq. (4)], momentum conservation [Eq. (3)], and the phonon dispersion relation [Eq. (5)], an emitted phonon satisfies ${ }^{18}$

$$
q=2\left(k \cos \theta-k_{s}\right),
$$

where $k_{s}=m s / \hbar$ is a characteristic wave vector. Equation (6) implies that the carrier velocity must be greater than the sound speed in order for Neganov-Luke phonons to be emitted. Additionally, $\theta<\pi / 2$ is required, implying that Neganov-Luke phonons are only emitted in the forward hemisphere relative to the electron wave vector. Note that the electron wave vector may deviate from the mean drift axis, so $\chi<\pi / 2$ is not required. A similar relation holds for phonon absorption, though we will find it unnecessary and so do not derive it.

The rate at which a carrier of energy $\epsilon$ emits (absorbs) a phonon of wave vector $\vec{q}$ per phonon wave vector mode is given by Fermi's Golden Rule (+ for emission, - for absorption)

$$
\Gamma( \pm \vec{q} ; \vec{k})=\frac{2 \pi}{\hbar}|\langle\vec{k} \mp \vec{q}|H| \vec{k}\rangle|^{2} \delta\left(\epsilon \mp h \nu-\epsilon^{\prime}\right),
$$

where $H$ is the deformation potential Hamiltonian, $\epsilon$ $=\hbar^{2} k^{2} / 2 m$ is the carrier kinetic energy before emission, and $\epsilon^{\prime}=\left(\hbar^{2}(\vec{k} \mp \vec{q})^{2} / 2 m\right)$ is the carrier kinetic energy after emission (absorption). The matrix elements are ${ }^{18}$

$$
\begin{aligned}
& |\langle\vec{k}-\vec{q}|H| \vec{k}\rangle|^{2}=\frac{E_{1}^{2} \hbar}{2 V \rho s} q\left(n_{q}+1\right), \\
& |\langle\vec{k}+\vec{q}|H| \vec{k}\rangle|^{2}=\frac{E_{1}^{2} \hbar}{2 \operatorname{V\rho s} q n_{q},}
\end{aligned}
$$

where $E_{1}$ is the deformation potential, $\rho$ is the mass density, $V$ is the semiconductor volume, and 
TABLE I. Ge and Si materials parameters and calculated distribution parameters. The electron and hole effective conduction mass $m$, the deformation potential $E_{1}$, the sound speed $s$, and the mass density $\rho$ are from (Ref. 18). $v_{d}, T_{c}$ and $\left\langle\left(v_{z}-v_{d}\right)^{2}\right\rangle^{1 / 2}$ are calculated in this paper at $300 \mathrm{~V} / \mathrm{m}$ for Ge and $400 \mathrm{~V} / \mathrm{m}$ for Si. $\left\langle\left(v_{z}\right.\right.$ $\left.\left.-v_{d}\right)^{2}\right\rangle^{1 / 2}=\sqrt{k_{B} T_{c} / m}$ is the standard deviation of the Gaussian thermal distribution corresponding to $T_{c}$.

\begin{tabular}{|c|c|c|c|c|}
\hline \multirow[b]{2}{*}{ Parameters } & \multicolumn{2}{|c|}{$\mathrm{Ge}$} & \multicolumn{2}{|c|}{$\mathrm{Si}$} \\
\hline & Electron & Hole & Electron & Hole \\
\hline$m$ (fraction of $m_{e}$ ) & 0.12 & 0.38 & 0.26 & 0.50 \\
\hline$E_{1}(\mathrm{eV})$ & 33.0 & 4.73 & 12.8 & 7.85 \\
\hline$s(\mathrm{~km} / \mathrm{s})$ & 5.40 & 5.40 & 9.15 & 9.15 \\
\hline$\rho\left(\mathrm{g} / \mathrm{cm}^{3}\right)$ & 5.32 & 5.32 & 2.33 & 2.33 \\
\hline$v_{d}(\mathrm{~km} / \mathrm{s})$ & 19.8 & 17.4 & 20.6 & 16.4 \\
\hline$T_{c}(\mathrm{~K})$ & 15.5 & 28.5 & 12.1 & 8.1 \\
\hline$\left\langle\left(v_{z}-v_{d}\right)^{2}\right\rangle^{1 / 2}(\mathrm{~km} / \mathrm{s})$ & 44.3 & 33.4 & 26.6 & 15.5 \\
\hline
\end{tabular}

$$
n_{q}=\frac{1}{e^{h \nu / k_{B} T}-1}
$$

is the thermal phonon distribution function in the semiconductor. $k_{B}$ is Boltzmann constant. At the $\sim 40 \mathrm{mK}$ operating temperature, the thermal phonon density at the frequencies of interest to us is negligible. Additionally, because the carriers drift more quickly than primary phonons propagate, the primary phonons generated at the particle interaction site are well behind the drifting carriers. Therefore, we take $n_{q} \approx 0$, thereby neglecting stimulated phonon emission and phonon absorption.

With the above approximations, and using Eqs. (4), (3), and (5) to rewrite the energy conservation $\delta$ function, the rate of phonon emission is

$$
\Gamma(\vec{q} ; \vec{k})=\frac{\pi}{\hbar^{2} k} \frac{E_{1}^{2} m}{V \rho s} \delta\left(\cos \theta-\cos \theta_{0}\right),
$$

with

$$
\cos \theta_{0}=\frac{q^{\prime}}{k}
$$

and

$$
q^{\prime}=\frac{q}{2}\left(1+\frac{2 k_{s}}{q}\right)
$$

\section{CARRIER DISTRIBUTIONS}

We have the probability for a single drifting electron in emitting a phonon but we still need to determine the electron distribution in k-space to calculate Neganov-Luke phonon distributions in frequency and in angle. The energy and moementum acquired by drifting carriers from the applied electric field is balanced by energy loss and momentum loss via the phonon emission calculated above, integrated over all emitted phonon wave vectors and all carrier energies. In this section, we use this overall conservations of energy and momentum requirements to determine the carrier distribution.

The theory of the carrier momentum distribution in an external electric field has been explored by Thornber and Feynman $^{19}$ and by Peeters and Devreese. ${ }^{20}$ The drifting carriers are not in thermal equilibrium with the semiconductor.
But statistically speaking, the carriers are in a quasi-steady state with an average momentum $\hbar \vec{k}_{d}=m \vec{v}_{d}$. When the drifting carrier density is low, the momentum-space distribution can be accurately described by a drifted Maxwellian distribution, ${ }^{17,21}$

$$
f\left(\vec{k} ; \vec{k}_{d}, T_{c}\right)=\left(\frac{h^{2}}{2 \pi m k_{B} T_{c}}\right)^{3 / 2} \exp \left(-\frac{\hbar^{2}\left(\vec{k}-\vec{k}_{d}\right)^{2}}{2 m k_{B} T_{c}}\right)
$$

where $\vec{k}_{d}$ is the carrier wave vector corresponding to drift with mean velocity $\vec{v}_{d}$ and $T_{c}$ is the carrier effective temperature. This distribution is centered at the average carrier momentum and has a width determined by the carrier effective temperature. Su et al. ${ }^{22}$ demonstrated from first principles the validity of an effective carrier temperature and derived a nonlinear relation between the carrier drift velocity and the applied electric field. Therefore, we take the drifted Maxwellian as a valid approximation.

The mobilities of the charge carriers in Si or Ge (Ref. 23 ) indicate that the carrier mean free path is a few micrometer in the electric field of $300 \mathrm{~V} / \mathrm{m}$ in $\mathrm{Ge}(400 \mathrm{~V} / \mathrm{m}$ in $\mathrm{Si})$ that we consider. The average carrier velocity in Table I is consistent with this. Therefore, the charge carrier interaction rate is high enough to ensure many interactions during drift across the detector. It is thus valid to assume randomization of the carrier wave vectors transverse to the drift direction for an ensemble of charge carriers created by a particle interaction. We could calculate the characteristic transverse extent of the electron distribution due to deformation potential phonon emission, but that is beyond the scope of this paper. For our purposes, we assume the transverse diffusion of the carriers remains small compared to the detector thickness so the transverse size of the distribution can be neglected.

With the drifted Maxwellian distribution in hand, let us relate the mean drift momentum and energy to the parameters of the distribution $k_{d}$ and $T_{c}$. For a carrier density $n_{0}$ in a volume $V$, the total number of carriers is $N=n_{0} V$. The carrier phase-space density is then $n\left(\vec{k} ; \vec{k}_{d}, T_{c}\right)$ $=n_{0} f\left(\vec{k} ; \vec{k}_{d}, T_{c}\right)$. We take the $z$-axis to be along $\vec{k}_{d}$. One can check that, for the drifted Maxwellian distribution, the mean $z$-axis projection of the carrier wave vector is indeed $k_{d}$ : 


$$
\hbar k_{d}=\frac{V}{N} \frac{2 \pi}{(2 \pi)^{3}} \int_{0}^{\infty} k^{2} d k \int_{0}^{\pi} \sin \alpha d \alpha n\left(\vec{k} ; \vec{k}_{d}, T_{c}\right) \hbar k \cos \alpha
$$

where an integral over carrier wave vector azimuthal angle $\beta$ has been done. Note that the factor of $V / N$ cancels the $n_{0}$ in $n\left(\vec{k} ; \vec{k}_{d}, T_{c}\right)$ : the mean momentum depends only on the shape of the distribution function.

The average carrier energy is also obtained by integrating over the drifted Maxwellian distribution:

$$
\bar{\epsilon}=\frac{V}{N} \frac{2 \pi}{(2 \pi)^{3}} \int_{0}^{\infty} k^{2} d k \int_{0}^{\pi} \sin \alpha d \alpha n\left(\vec{k} ; \vec{k}_{d}, T_{c}\right) \frac{\hbar^{2} k^{2}}{2 m} .
$$

We obtain

$$
\bar{\epsilon}=\frac{3}{2} k_{B} T_{c}+\frac{\hbar^{2} k_{d}^{2}}{2 m} .
$$

Again, the factor of $V / N$ cancels the $n_{0}$ in the phase-space distribution function so that the mean energy depends only on the shape of the distribution function.

We may now integrate the phonon emission rate over the drifted Maxwellian distribution. The emission rate in a given phonon mode with a wave vector $\vec{q}$ defined by polar angle $\chi$ and azimuthal angle $\delta$, integrated over the carrier phase space distribution (i.e., over real space volume and wave vector space volume) is

$$
\begin{aligned}
& \Gamma\left(\vec{q} ; k_{d}, T_{c}\right) \\
& =\frac{n_{0} V}{(2 \pi)^{3}} \int_{k_{0}}^{\infty} k^{2} d k \int_{0}^{\pi} \sin \alpha d \alpha \int_{0}^{2 \pi} d \beta \Gamma(\vec{q} ; \vec{k}) f\left(\vec{k} ; \vec{k}_{d}, T_{c}\right),
\end{aligned}
$$

where $k_{0}=(q / 2)+k_{s}$ is the smallest carrier wave vector capable of emitting phonons. The result is of course parametrically dependent on $k_{d}$ and $T_{c}$. We have dropped the vector nature of $\vec{k}_{d}$ by taking the phonon wave vector to be in a coordinate system whose $z$-axis is the mean drift direction, $\vec{k}_{d}$.

We can perform this integral by changing coordinate systems. Let us take the emitted phonon direction to be along the new $z$-axis. The carrier thus has polar angle $\theta$ as defined earlier. Let the carrier's new azimuthal angle be $\eta$. The integral thus becomes

$$
\begin{aligned}
& \Gamma\left(\vec{q} ; k_{d}, T_{c}\right)=\frac{N}{(2 \pi)^{3}}\left(\frac{h^{2}}{2 \pi m k_{B} T_{c}}\right)^{3 / 2} \int_{k_{0}}^{\infty} k^{2} d k \int_{-1}^{1} d \cos \theta \\
& \int_{0}^{2 \pi} d \eta \Gamma(\vec{q} ; \vec{k}) \exp \left(-\frac{\hbar^{2} k_{d}^{2}+\hbar^{2} k^{2}-2 \hbar^{2} k k_{d} \cos \alpha}{2 m k_{B} T_{c}}\right),
\end{aligned}
$$

where $\cos \alpha=\cos \chi \cos \theta-\sin \chi \sin \theta \cos \eta$ is obtained by spherical angle addition.

Since the carriers are assumed to have a steady state momentum distribution, the force exerted by the drift field on the ensemble of carriers must be equal to the rate of $z$-axis momentum emission in phonons:

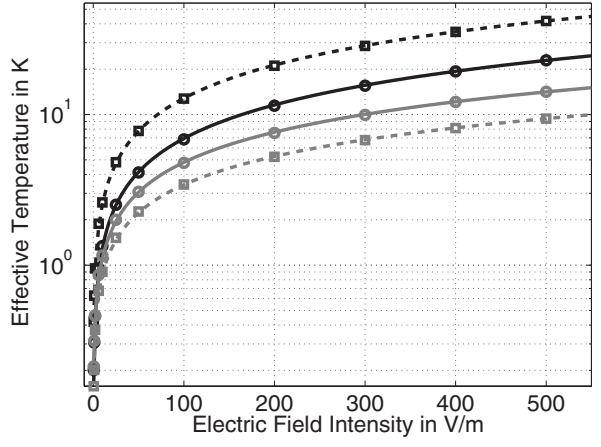

FIG. 2. The electron (hole) effective temperature as a function of electric field strength. Circles: electrons. Squares: holes. Black: Ge (300 V/m). Gray: Si $(400 \mathrm{~V} / \mathrm{m})$. The lines are power law fits.

$$
N e E=\frac{V}{(2 \pi)^{3}} 2 \pi \int_{0}^{\infty} q^{2} d q \int_{-1}^{1} d \cos \chi \Gamma\left(\vec{q} ; k_{d}, T_{c}\right) \hbar q \cos \chi,
$$

where $E$ is the magnitude of the external electric field $\vec{E}$. We have assumed that, once one integrates over carrier wave vectors, the integrand can no longer depend on the phonon azimuthal angle $\delta$ relative to the mean drift direction, and so we have integrated over it. Also, recall that $\Gamma\left(\vec{q} ; k_{d}, T_{c}\right)$ contains a factor $N$ that cancels the $N$ on the left side [Eq. (19)] and a factor of $\Gamma(\vec{q} ; \vec{k})$ which contains a factor of $1 / V[\mathrm{Eq}$. (11)] that cancels the $V$ prefactor.

Similarly, the rate of work done on the carrier distribution by the external field must equal the rate of energy loss by phonon emission:

$$
N e E v_{d}=\frac{V}{(2 \pi)^{3}} 2 \pi \int_{0}^{\infty} q^{2} d q \int_{-1}^{1} d \cos \chi \Gamma\left(\vec{q} ; k_{d}, T_{c}\right) h \nu,
$$

where we have used the linear dispersion relation $h \nu=\hbar s q$. The same notes about $\delta$ and the $N$ and $V$ factors apply.

We thus have two nonlinear equations that can be solved for $v_{d}$ and $T_{c}$. (Recall, $\hbar k_{d}=m v_{d}$.) In practice, we obtain this solution by calculating, for candidate $v_{d}$ and $T_{c}$, electric field magnitudes $E^{m}$ from Eq. (20) and $E^{e}$ from Eq. (21), and then minimizing $\chi^{2}=\left(1-E^{m} / E\right)^{2}+\left(1-E^{e} / E\right)^{2}$ with respect to $v_{d}$ and $T_{c}$. (An input value of $E$ is required; otherwise, $E$ would be a free parameter also.) We consider the minimization to have converged when $\chi^{2}<10^{-6}$. Table I summarizes the semiconductor materials parameters we have used, the resulting $v_{d}$ and $T_{c}$ for electrons and holes, and the standard deviation of the $z$ component of the drift velocity at typical bias fields for the CDMS detectors $(300 \mathrm{~V} / \mathrm{m}$ for $\mathrm{Ge}, 400 \mathrm{~V} / \mathrm{m}$ for $\mathrm{Si})$.

Both $v_{d}$ and $T_{c}$ depend on materials parameters such as effective mass $m$, deformation potential $E_{1}$, sound speed $s$, and semiconductor mass density $\rho$, as well as on the applied electric field strength $E$. Figures 2 and 3 show the electron and hole effective temperatures and drift velocities as a function of the external electric field. We make power law $\left(T_{c}\right.$ $=b_{1} E^{a_{1}}+c_{1}, v_{d}=b_{2} E^{a_{2}}+c_{2}$ ) fits to the relations and list the fit parameters in Table II. 


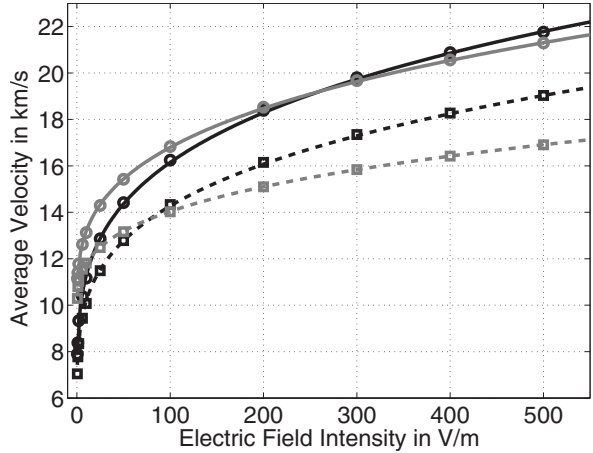

FIG. 3. The electron (hole) average drift velocity as a function of electric field strength. Circles: electrons. Squares: holes. Black: Ge (300 V/m). Gray: $\mathrm{Si}(400 \mathrm{~V} / \mathrm{m})$. The lines are power law fits.

We note that, because thermal phonons are not included in this calculation, our predictions may become increasingly incorrect at low electric field strength.

\section{NEGANOV-LUKE PHONON DISTRIBUTIONS}

With the distribution function $\Gamma\left(\vec{q} ; k_{d}, T_{c}\right)$ of the emitted Neganov-Luke phonons, we can calculate the angular and frequency distributions of Neganov-Luke phonon power and total emitted power within our drifted Maxwellian approximation. We will consider various integrals of $P_{E}\left(\vec{q} ; k_{d}, T_{c}\right)$ $=\Gamma\left(\vec{q} ; k_{d}, T_{c}\right) h \nu / N$, which is the power emitted in the phonon wave vector mode $\vec{q}$ by a drifted Maxwellian distribution parameterized by $k_{d}$ and $T_{c}$, per carrier.

The Neganov-Luke phonon power angular distribution function per carrier is defined to be $P_{E}(\chi)$ (recall $\chi$ is the polar angle with respect to the drift direction as indicated in Fig. 1) and is obtained by integrating $P_{E}\left(\vec{q} ; k_{d}, T_{c}\right)$ over phonon wave vector azimuthal angle and magnitude:

$$
\begin{aligned}
P_{E}(\chi) & =\frac{1}{N} \frac{V}{(2 \pi)^{3}} 2 \pi \int_{0}^{\infty} q^{2} d q \Gamma\left(\vec{q} ; k_{d}, T_{c}\right) h \nu \\
& =\frac{\hbar s}{N} \frac{V}{(2 \pi)^{2}} \int_{0}^{\infty} q^{3} d q \Gamma\left(\vec{q} ; k_{d}, T_{c}\right),
\end{aligned}
$$

where we have used $h \nu=\hbar s q$. It has units of power per unit polar angle $d \chi$. Note that the $V / N$ factor is canceled by a factor of $N$ in the definition of $\Gamma\left(\vec{q} ; k_{d}, T_{c}\right)$ (Eq. (19)) and by a factor of $1 / V$ in the definition of $\Gamma(\vec{q} ; \vec{k})$ (Eq. (11)), which is a factor in $\Gamma\left(\vec{q} ; k_{d}, T_{c}\right)$ (Eq. (19), again).

TABLE II. Parameters of power law fits to carrier effective temperature and average drift velocity as a function of applied electric field.

\begin{tabular}{lccccc}
\hline \hline \multirow{2}{*}{ Parameters } & \multicolumn{2}{c}{ Ge } & & \multicolumn{2}{c}{$\mathrm{Si}$} \\
\cline { 2 - 3 } \cline { 5 - 6 } \cline { 5 - 6 } & Electron & Hole & & Electron & Hole \\
\hline$a_{1}$ & 0.7450 & 0.7470 & & 0.6800 & 0.6300 \\
$b_{1}$ & 0.2226 & 0.4001 & & 0.2047 & 0.1860 \\
$c_{1}$ & 0.0500 & 0.2300 & & 0.1240 & 0.0500 \\
$a_{2}$ & 0.2620 & 0.2670 & & 0.3020 & 0.3150 \\
$b_{2}$ & 3216 & 2594 & & 1793 & 1010 \\
$c_{2}$ & 5400 & 5400 & & 9500 & 9500 \\
\hline \hline
\end{tabular}

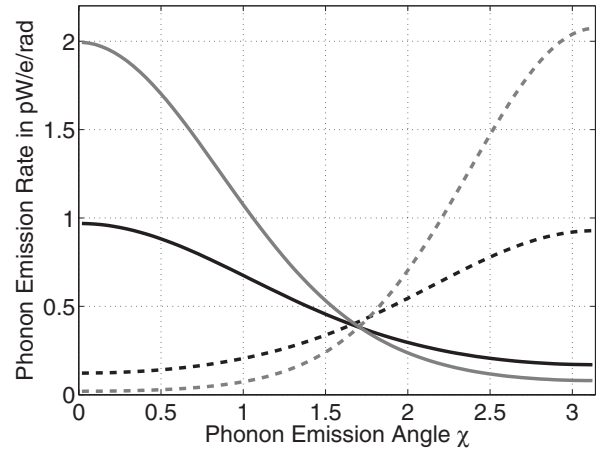

FIG. 4. The Neganov-Luke phonon power angular distribution function. Solid lines: electrons. Dashed lines: holes. Black: Ge $(300 \mathrm{~V} / \mathrm{m})$. Gray: $\mathrm{Si}$ $(400 \mathrm{~V} / \mathrm{m})$.

The Neganov-Luke phonon power emitted per carrier and per unit phonon frequency interval as a function of phonon frequency is obtained by integrating $P_{E}\left(\vec{q} ; k_{d}, T_{c}\right)$ over phonon solid angle, including a $q^{2}$ factor from the phonon wave vector magnitude differential:

$$
\begin{aligned}
P_{E}(\nu) & =\frac{2 \pi}{s} \frac{1}{N} \frac{V}{(2 \pi)^{3}} 2 \pi \int_{0}^{\pi} \sin \chi d \chi q^{2} \Gamma\left(\vec{q} ; k_{d}, T_{c}\right) h \nu \\
& =\frac{\hbar}{N} \frac{V}{2 \pi} \int_{0}^{\pi} \sin \chi d \chi q^{3} \Gamma\left(\vec{q} ; k_{d}, T_{c}\right),
\end{aligned}
$$

where we have inserted a factor of $2 \pi / s$ to convert from per wave vector interval $d q$ to per frequency interval $d \nu$ and we have again used $h \nu=\hbar s q$. It has units of power per unit phonon frequency interval. The $V / N$ factor is canceled in similar fashion.

The total Neganov-Luke phonon power emitted per carrier is obtained by integrating $P_{E}\left(\vec{q} ; k_{d}, T_{c}\right)$ over phonon wave vector in all three dimensions:

$$
\begin{aligned}
P_{E} & =\frac{1}{N} \frac{V}{(2 \pi)^{3}} 2 \pi \int_{0}^{\infty} q^{2} d q \int_{0}^{\pi} \sin \chi d \chi \Gamma\left(\vec{q} ; k_{d}, T_{c}\right) h \nu \\
& =\frac{\hbar s}{N} \frac{V}{(2 \pi)^{2}} \int_{0}^{\infty} q^{3} d q \int_{0}^{\pi} \sin \chi d \chi \Gamma\left(\vec{q} ; k_{d}, T_{c}\right),
\end{aligned}
$$

where, again, $V / N$ is canceled in similar fashion.

Figures 4 and 5 show the Neganov-Luke phonon power

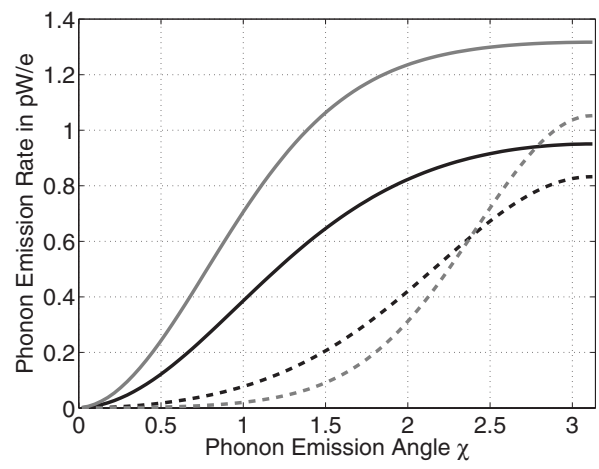

FIG. 5. The cumulative integral of the Neganov-Luke phonon power angular distribution. Solid lines: electrons. Dashed lines: holes. Black: Ge (300 V/m). Gray: Si $(400 \mathrm{~V} / \mathrm{m})$. 


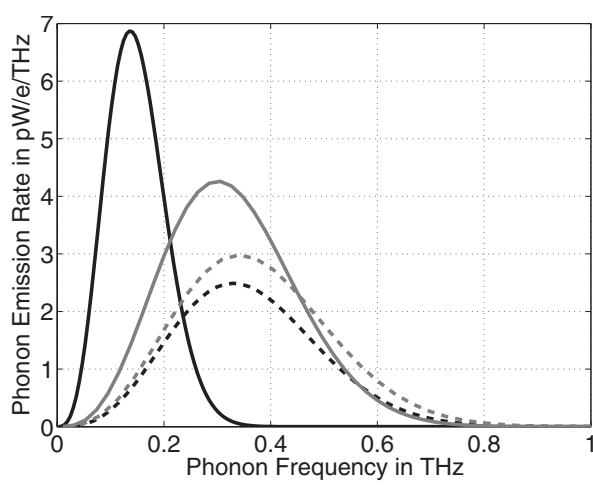

FIG. 6. The Neganov-Luke phonon power frequency distribution. Solid lines: electrons. Dashed lines: holes. Black: Ge (300 V/m). Gray: Si (400 $\mathrm{V} / \mathrm{m})$.

angular distribution function $P_{E}(\chi)$ and its cumulative integral $\int_{0}^{\chi} P_{E}\left(\chi^{\prime}\right) \sin \left(\chi^{\prime}\right) d \chi^{\prime}$ for Ge at $300 \mathrm{~V} / \mathrm{m}$ and for $\mathrm{Si}$ at 400 $\mathrm{V} / \mathrm{m}$. The $z$ direction is defined to be the electron drift direction. Holes drift to $-z . P_{E}(\chi<\pi / 2) / P_{E}(\chi>\pi / 2)$ is approximately $2.4(4.8)$ for electrons in $\mathrm{Ge}(\mathrm{Si})$, while $P_{E}(\chi$ $>\pi / 2) / P_{E}(\chi<\pi / 2)$ is approximately $2.7(9.0)$ for holes in $\mathrm{Ge}(\mathrm{Si})$. The Neganov-Luke phonons are thus more asymmetrically distributed in $\mathrm{Si}$ than in Ge, favoring the carrier drift direction. This occurs because the carrier effective temperature is smaller in Si than in Ge, as indicated in Table I.

Figures 6 and 7 show the emitted Neganov-Luke phonon power frequency distribution and its cumulative integral $\int_{0}^{\nu} P_{E}\left(\nu^{\prime}\right) d \nu^{\prime}$ for Ge at $300 \mathrm{~V} / \mathrm{m}$ and for $\mathrm{Si}$ at $400 \mathrm{~V} / \mathrm{m}$. The Neganov-Luke phonon power peaks at $0.14 \mathrm{THz}(0.33 \mathrm{THz})$ for electrons (holes) in Ge and at $0.31 \mathrm{THz}(0.34 \mathrm{THz})$ for electrons (holes) in $\mathrm{Si}$. Of relevance to the operation of CDMS detectors is the fraction of Neganov-Luke phonons above $0.084 \mathrm{THz}(0.34 \mathrm{meV})$, the energy required to break Cooper pairs in superconducting aluminum, $2 \Delta_{A l}$. These fractions are $91.5 \%$ (99.7\%) for electrons (holes) in Ge and 99.7\% (99.9\%) for electrons (holes) in $\mathrm{Si}$.

We emphasize that our numerical results are calculated in the context of our model, which considers only acoustic phonons in a homogeneous medium. Numerical results may change in a more sophisticated model, though we expect that our qualitative conclusions will continue to hold.

We have observed that, in Ge, the Neganov-Luke phonon distribution emitted from electrons is lower in frequency

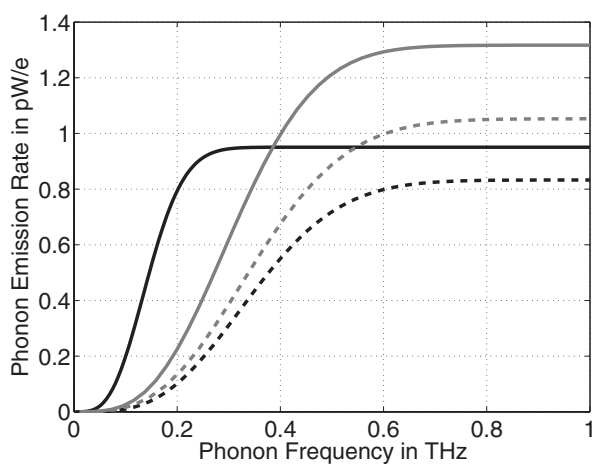

FIG. 7. The cumulative integral of the Neganov-Luke phonon power frequency distribution. Solid lines: electrons. Dashed lines: holes. Black: Ge $(300 \mathrm{~V} / \mathrm{m})$. Gray: Si $(400 \mathrm{~V} / \mathrm{m})$. than from holes, and that Neganov-Luke phonons are emitted primarily in the forward hemisphere of carrier drift. In the absence of frequency down-conversion, these facts have no implications for Neganov-Luke phonon collection because all phonons eventually reach the superconducting aluminum phonon absorbers on the grounded detector face. However, when Neganov-Luke phonon frequency downconversion, which is due to diffusive reflection ${ }^{24-28}$ at the surface as well as due to anharmonic decay and isotope (impurity) scattering ${ }^{29,30}$ in the bulk, is considered, the path and path length to reach the phonon sensors become important. In particular, since there is an appreciable difference in the phonon power distribution as a function of frequency between electrons and holes in Ge, with electrons emitting phonons weighted toward lower frequencies than holes, phonons emitted by electrons are more susceptible to downconversion to energies below the aluminum pair-breaking energy. Thus, we expect that the collectable fraction of phonon power emitted by electrons will be greater when they drift toward the phonon sensors, while the collectable fraction of phonon power emitted by holes will be much less dependent on drift direction. Therefore, when a negative bias is applied, causing electrons to drift toward the phonon sensor, a larger fraction of Neganov-Luke phonon energy should be collected. By comparison, there should be no such effect in $\mathrm{Si}$ because the phonon power distributions as a function of frequency for electrons and holes are much more similar.

To summarize, we have analyzed the physics of Neganov-Luke phonon emission from the drifting charge carriers in an external electric field applied to semiconducting substrates at sub-kelvin temperatures. In the drifted Maxwellian approximation, we have calculated the average carrier drift velocities, the effective temperatures, and the angular and frequency distributions of emitted NeganovLuke phonon power for typical electric fields. Such phonons are more forward-directed in Si than in Ge. Most of the Neganov-Luke phonon power is collectable by the superconducting aluminum phonon absorbers employed in the CDMS detectors. However, a dependence of the efficiency collecting these phonons in $\mathrm{Ge}$ on the bias polarity is expected. For holes in Ge, more advanced Monte Carlo analyses show that optical phonon emission is important and should be included in more accurate calculations. ${ }^{31}$

The Neganov-Luke phonon power distributions are critical for an understanding and improving event position reconstruction in the CDMS detectors. Future work can use these distributions, combined with phonon propagation, decay, and reflection, to model the position dependence of the phonon signal in CDMS detectors.

\section{ACKNOWLEDGMENTS}

The author thanks Blas Cabrera, Sunil Golwala, and Daniel Akerib for stimulating discussion and for support for this analysis. The work was done while author was at Case Western Reserve University, California Institute of Technology, and Argonne National Laboratory. The work was supported by the National Science Foundation under Grant Nos. AST-9978911 and PHY-9722414, by the Department of En- 
ergy under Contract Nos. DE-AC03-76SF00098, DE-FG0390ER40569, DE-FG03-91ER40618, and DE-FG0294ER40823, by FNAL, operated by the Universities Research Association, Inc., under Contract No. DE-AC0276CH03000 with the Department of Energy, and by the California Institute of Technology. The work at Argonne National Laboratory was supported by Office of Science and Office of Basic Energy Sciences of the U.S. Department of Energy, under Contract No. DE-AC02-06CH11357.

${ }^{1}$ G. Steigman and M. S. Turner, Nucl. Phys. B 253, 375 (1985).

${ }^{2}$ B. W. Lee and S. Weinberg, Phys. Rev. Lett. 39, 165 (1977).

${ }^{3}$ S. Weinberg, Phys. Rev. Lett. 48, 1303 (1982).

${ }^{4}$ G. Jungman, M. Kamionkowski, and K. Griest, Phys. Rep. 267, 195 (1996).

${ }^{5}$ G. Bertone, D. Hooper, and J. Silk, Phys. Rep. 405, 279 (2005).

${ }^{6}$ D. S. Akerib, J. Alvaro-Dean, M. S. Armel-Funkhouser, M. J. Attisha, L. Baudis, D. A. Bauer, J. Beaty, P. L. Brink, R. Bunker, S. P. Burke, et al., Phys. Rev. Lett. 93, 211301 (2004).

${ }^{7}$ D. S. Akerib, M. J. Attisha, C. N. Bailey, L. Baudis, D. A. Bauer, P. L. Brink, P. P. Brusov, R. Bunker, B. Cabrera, D. O. Caldwell, et al., Phys. Rev. Lett. 96, 011302 (2006).

${ }^{8}$ Z. Ahmed, D. S. Akerib, S. Arrenberg, C. N. Bailey, L. Baudis, D.A. Bauer, J. Beaty, P. L. Brink, T. Bruch, et al., Phys. Rev. Lett. 102, 011301 (2009).

${ }^{9}$ R. M. Clarke, P. L. Brink, B. Cabrera, P. Colling, M. B. Crisler, A. K. Davies, S. Eichblatt, R. J. Gaitskell, J. Hellmig, J. M. Martinis, S. W. Nam, T. Saab, and B. A. Young, Appl. Phys. Lett. 76, 2958 (2000).

${ }^{10}$ D. S. Akerib, M. S. Armel-Funkhouser, M. J. Attisha, C. N. Bailey, L.
Baudis, D. A. Bauer, P. L. Brink, R. Bunker, B. Cabrera, D. O. Caldwell, et al., Phys. Rev. D 72, 052009 (2005).

${ }^{11}$ B. Neganov and V. Trofimov, Otkrytiya, Izobret. 146, 215 (1985).

${ }^{12}$ P. N. Luke, J. Appl. Phys. 64, 6858 (1988).

${ }^{13}$ H. J. Maris, Phys. Rev. B 41, 9736 (1990).

${ }^{14}$ S. Tamura, J. A. Shields, and J. P. Wolfe, Phys. Rev. B 44, 3001 (1991)

${ }^{15}$ M. E. Msall and J. P. Wolfe, Phys. Rev. B 56, 9557 (1997).

${ }^{16}$ C. Kittel, Introduction To Solid State Physics (Wiley, New York, 1976).

${ }^{17}$ K. Seeger, Semiconductor Physics: An Introduction (Springer-Verlag, New York, 2004).

${ }^{18}$ B. Cabrera, Electron Phonon Scattering, note93, CDMS note, 2003.

${ }^{19}$ K. K. Thornber and R. P. Feynman, Phys. Rev. B 1, 4099 (1970).

${ }^{20}$ F. M. Peeters and J. T. Devreese, Phys. Rev. B 23, 1936 (1981).

${ }^{21}$ B. K. Ridley, Quantum Processes in Semiconductors (Oxford University Press, Oxford, England, 1999).

${ }^{22}$ Z. B. Su, L. Y. Chen, and C. S. Ting, Phys. Rev. B 38, 3866 (1988).

${ }^{23}$ Handbook Series on Semiconductor Parameters, edited by M. Levinshtein, S. Rumyantsev, and M. Shur (World Scientific, Singapore, 1996), Vol. 1.

${ }^{24}$ F. Gramatica and E. Jeannet, Europhys. Lett. 10, 111 (1989).

${ }^{25}$ S. Burger, K. Lassmann, and W. Eisenmenger, J. Low Temp. Phys. 61, 401 (1985).

${ }^{26}$ J. Mebert and W. Eisenmenger, Z. Phys. B 95, 231 (1994).

${ }^{27}$ T. Klitsner and R. O. Pohl, Phys. Rev. B 36, 6551 (1987).

${ }^{28}$ M. N. Wybourne, C. G. Eddison, and M. J. Kelly, J. Phys. C 17, L607 (1984).

${ }^{29}$ H. J. Maris, J. Low Temp. Phys. 93, 355 (1993).

${ }^{30}$ Y. B. Levinson, "Phonon Propagation with Frequency Down-conversion" in Nonequilibrium Phonons in Nonmetallic Crystals, edited by W. Eisenmenger and A. A. Kaplyanskii (Elsevier Science, Amsterdam, 1986).

${ }^{31}$ K. M. Sundqvist and B. Sadoulet, J. Low Temp. Phys. 151, 443 (2008). 